\title{
El cuerpo como objeto de deseo en obesos mórbidos con antecedentes de abuso sexual.
}

The body as an object of desire in morbidly obese patients with a history of sexual abuse.

\author{
Begoña Mazagatos ${ }^{a}$, Sandra Ingles-Borda ${ }^{\text {b }}$,Amanda López-Picado ${ }^{\mathrm{c}}$. \\ ${ }^{a, b}$ Servicio de Psiquiatría. Hospital Universitario Araba. Vitoria-Gasteiz, España. ${ }^{a}$ Psicoanalista miembro \\ de la Asociación Psicoanalítica de Madrid perteneciente a la IPA (International Psychoanalytical Associa- \\ tion), España. ${ }^{c}$ Unidad de Investigación de Araba. Hospital Universitario Araba. Vitoria-Gasteiz, España.
}

Correspondencia: Amanda López-Picado (Amanda.lopezpicado@osakidetza.net)

Recibido: 03/04/2014; aceptado: 06/07/2014

RESUMEN: Introducción: La imagen corporal del obeso mórbido puede ser usada como factor de protección frente a la sexualidad. Se ha hipotetizado que los sujetos con antecedentes de abuso sexual serían más proclives a utilizar la obesidad mórbida en este sentido. Acercarnos a la verificación de esta hipótesis en una muestra de 398 pacientes con obesidad mórbida que solicitan intervención de cirugía bariátrica.

Método: Entrevista semiestructurada con recogida sistemática de datos, de los abusos sufridos y de las vivencias y repercusiones de la obesidad en las relaciones sexuales de los pacientes.

Resultados: la mayoría de los pacientes entrevistados con antecedentes de abusos sexuales eran mujeres $(n=39 ; 88,6 \%)$. Los pacientes con antecedentes de abusos sexuales presentaban una comorbilidad psiquiátrica mayor y menor autoestima (2.60 OR $(1,32-5,13))$ aunque la obesidad no representaba un problema en sus relaciones sexuales $1.52(0,71-3,24))$.

Conclusión: La utilización de la imagen corporal del obeso como barrera de protección ante la sexualidad no requiere del antecedente de abuso sexual.

PALABRAS CLAVE: imagen corporal, obesidad mórbida, abusos sexuales.
ABSTRACT: Background: Morbid obesity may use their body image in order to avoid attracting erotic attention. Some authors have suggest that people who have been sexually abused are likely to used their body image in that way. We try to attempt to verify this hypothesis in a sample of 398 morbidly obese patients who requested bariatric surgery. Method: A semi-structured interview with systematic collection of information regarding the abuse suffered and the experiences and repercussions of obesity on the patients' sexual relations.

Results: The majority of patients interviewed with a history of abuse were women $(n=39 ; 88.6 \%)$. Patients with a history of sexual abuse presented higher psychiatric comorbidity and lower self-esteem (2.60 (1.32-5.13)), although the belief and convictions of interference of their body image in their sexuality was similar to those who had not suffered sexual abuse (1.52 (0.71-3.24)).

Conclusion: The use of an obese subject's body image as a protective barrier against sexuality does not require a history of sexual abuse.

KEY WORDS: body image, morbid obesity, sexual abuse.

\section{Introducción}

La obesidad mórbida es una patología cada vez más frecuente en nuestro entorno. Hay múltiples factores socioculturales, interpersonales, intrapsiquicos y orgánicos, incluido los genéticos, que determinan y/o condicionan la evolución hacia la obesidad mórbida, aunque aún no ha sido posible establecer de forma fehaciente el impacto ni la forma de interacción entre cada uno de ellos en la génesis de la obesidad mórbida (1-5).

Los aspectos psicosociales de esta enfermedad son especialmente importantes, no sólo por cuanto se considera su calidad de vida cuando ya existe la obesi- 
dad severa sino por la posible relación de aspectos socioculturales y dificultades emocionales durante la infancia, que habrían influido tanto en la adquisición de comportamientos y hábitos de alimentación no saludables, como en el propio metabolismo (1).

Entre ellos se han descrito los maltratos y abusos sexuales infantiles, que parecen contribuir en el desarrollo de la obesidad, aunque no específicamente en la obesidad mórbida (6-14).

Existen diferentes abordajes desde lo sociocultural e intrapsíquico hasta lo neurobiológico para acercarse a entender esta interacción.

Desde la neurobiología la vivencia del maltrato infantil implica la exposición del cerebro en desarrollo a un estrés crónico y/o grave, ocasionando la hiperactivación del eje hipotalámico-hipófisis suprarrenal (15), provocando una alteración anatomofuncional del sistema límbico que generaría niveles elevados de cortisol, lo que aumenta la capacidad de depósito de grasa en los adipositos (16) y contribuiría así al desarrollo de la obesidad $(8,17)$.

Desde la perspectiva sociocultural, el modelo erótico actual no coincide con la imagen del obeso (18-20), en este sentido, Glinski (21) se pregunta si la obesidad podría actuar como elemento de protección ante las relaciones interpersonales estrechas y Näslund (22) considera como datos de mal pronóstico tras cirugía bariatrica, los antecedentes de abusos sexuales en la infancia. Siguiendo en esta línea argumental las personas que han sido objeto de abuso sexual es previsible que tuvieran, en general, una actitud más desconfiada frente a la sexualidad y en algunos casos de obesidad mórbida podrían utilizar su imagen corporal para no ser reclamo erótico (1).

En este trabajo, al igual que los de otros autores $(19,23)$, analiza la relación entre la obesidad y la erotización.

Conscientes de la complejidad del tema nos planteamos en este trabajo la descripción de los obesos mórbidos, de nuestra muestra, que refieren antecedentes de abusos sexuales, y estudiamos si existe relación entre estos antecedentes y sus vivencias de la repercusión de la obesidad en sus relaciones sexuales y así mismo exploramos las características de estos abusos.

\section{Material y Métodos}

\section{Diseño}

Estudio prospectivo observacional dentro de la práctica clínica habitual en una muestra consecutivo de pacientes. Se estudian 393 pacientes con obesidad mórbida que solicitan intervención de cirugía bariátrica en Hospital Universitario 
ORIGINALES Y REVISIONES

de Álava, sede Txagorritxu de Vitoria-Gasteiz (Spain) entre marzo de 2001 y junio de 2010. De estos un total de $44(11,2 \%)$ pacientes presentaban historia de abuso sexual.

\section{Método:}

Entrevista psiquiátrica semiestructurada encuadrada en la práctica clínica habitual de evaluación de los pacientes con obesidad mórbida que solicitan tratamiento quirúrgico. Donde se recogen entre otros datos, a los que hacemos referencia en este artículo: características sociodemográficas, índice de masa corporal (IMC), comorbilidad psiquiátrica (tratamiento psiquiátrico o uso de psicótropos), antecedentes de abusos sexuales y características de estos:(edad, frecuencia, si se realizó por parte de un familiar/allegado o extraño y si hubo ocultismo o no, disminución de autoestima y si hubo cambios en el entorno como consecuencia) y repercusión de la obesidad en sus relaciones sexuales y a nivel psicosocial. Todas las entrevistas fueron realizadas por una única entrevistadora.

Consideramos como abusos sexuales solo los que el paciente refiere como tales siendo preguntados explícitamente por ello en un momento emocional concreto de la entrevista, cuando está hablando de su biografía. Esto indica que el hecho fue vivido por el paciente como erotizado e impuesto, lo que nos parece más significativo para las repercusiones psicológicos que si lo definiéramos de acuerdo a datos formales externos.

En cuanto a las relaciones sexuales, les preguntamos específicamente si su obesidad les repercute de alguna forma en sus relaciones sexuales y como. Las respuestas las agrupamos en 5 apartados: dificultad de movimientos, vergüenza del propio aspecto, ninguna repercusión, no interés en relaciones y no pareja (incluido esporádicas). Las 2 últimas categorías, es decir, no interés en relaciones o no tener o haber tenido pareja siendo obeso, lo recogimos y no se utilizaron para el análisis ya que en estos casos no se puede constatar la interferencia de la obesidad en las relaciones. Corresponderían al clásico apartado "no sabe, no contesta".

\section{Análisis estadístico}

Las variables continuas, como la edad y el índice de masa corporal, se describieron mediante su media, desviación estándar, mientras que, las variables categóricas se describieron mediante la distribución de frecuencias y porcentual correspondiente. La comparación de la edad y el IMC entre los pacientes con antecedentes de abusos sexuales y los pacientes sin dichos antecedentes se realizó mediante la prueba $\mathrm{T}$ Student mientras que la comparación de las restantes variables (estado civil, nivel de estudios, uso de psicótropos y repercusión en las relaciones 
sexuales entre otras) se utilizó la prueba de Chi cuadrado. En todo el análisis un valor de $\mathrm{p}<0,05$ fue considerado significativo. El estudio estadístico consta del análisis descriptivo de las variables mediante SPSS v.18.

\section{Resultados}

La muestra global se compone de 393 pacientes de una edad de $41,2 \pm 11,0$, con un IMC de 48,4 $\pm 5,5$ (rango de 35,0-65,8), predominio de sexo femenino $76,3 \%$ $(\mathrm{n}=300)$, bajo nivel educativo, siendo un $64 \%$ los que tenían menos de 10 años de educación formal. De este grupo, $44(11,2 \%)$ pacientes presentaban antecedentes de abuso sexual. Las características sociodemográficas se muestran en la tabla1, sin que hubiera diferencias estadísticamente significativas entre los pacientes con antecedentes de abusos y entre los que no excepto en el sexo, ya que la mayoría de los abusados eran mujeres $(88,6 \% ; n=39)$.

Tabla1.

Datos demográficos de los 2 grupos de pacientes:

\begin{tabular}{|c|c|c|c|c|}
\hline & & Grupo abuso sexual $(n=44)$ & $\begin{array}{l}\text { Grupo no abuso sexual } \\
(n=349)\end{array}$ & $\mathbf{p}$ \\
\hline \multirow{2}{*}{ Sexo } & Hombres & $5(11,4 \%)$ & $88(25,2 \%)$ & \multirow{2}{*}{0,042} \\
\hline & Mujeres & $39(88,6 \%)$ & $261(74,8 \%)$ & \\
\hline \multicolumn{2}{|l|}{ Edad } & $46,0 \pm 13,2$ & $46,2 \pm 11,3$ & 0,904 \\
\hline \multirow[b]{2}{*}{ Estudios } & Primarios & $27(61,4 \%)$ & $225(64,7 \%)$ & \multirow[b]{2}{*}{0,739} \\
\hline & $\begin{array}{l}\text { Secundarios o } \\
\text { Superiores }\end{array}$ & $17(38,6 \%)$ & $123(35,3 \%)$ & \\
\hline \multirow{2}{*}{ Estado civil } & Con pareja & $14(31,8 \%)$ & $225(64,5 \%)$ & \multirow{2}{*}{0,738} \\
\hline & Sin pareja & $30(68,2 \%)$ & $124(35,5 \%)$ & \\
\hline \multirow{2}{*}{ Situación laboral } & Activo & $17(38,6 \%)$ & $177(50,7 \%)$ & \multirow{2}{*}{0,151} \\
\hline & Parado & $27(61,4 \%)$ & $155(49,3 \%)$ & \\
\hline \multicolumn{2}{|l|}{ IMC } & $44,8 \pm 5,5$ & $46,2 \pm 5,6$ & 0,120 \\
\hline
\end{tabular}

IMC: índice de masa corporal

Describiendo las características de los abusos sexuales, se encontró que el $68,2 \%(n=33)$ habían sufrido abusos por parte de un familiar, el 70,5\%(n=31) había ocultado el abuso y la edad media de cuando sufrieron los abusos fue entorno a los 10 años (media $9,9 \pm 5,8$ ).

Dentro del grupo con antecedentes de abuso sexual, el $68,2 \%(\mathrm{n}=30)$ requirieron tratamiento psiquiátrico previo a la intervención, respecto al $31,8 \%(\mathrm{n}=14)$ que no recibieron tratamiento (OR:3,12 $(1,60-6,10))$. Entre aquellos que han sufri- 
do abusos sexuales, el 70,5\% $(\mathrm{n}=31)$ refieren disminución de la autoestima por el hecho mismo de la obesidad, en comparación el 29,5\% $(\mathrm{n}=13)$ que no (OR:2,60 $(1,32-5,13))$.

El $62,5 \%(n=20)$ de los que han tenido abuso sexual presentaban repercusiones en las relaciones sexuales por la obesidad, frente al 37,5\% (n=12) de los abusado que no constaron este hecho pero la diferencia no es estadísticamente significativa (OR:1,52 (0,71-3,24)). (Ver tabla 2).

Tabla2.

Repercusiones del abuso sexual:

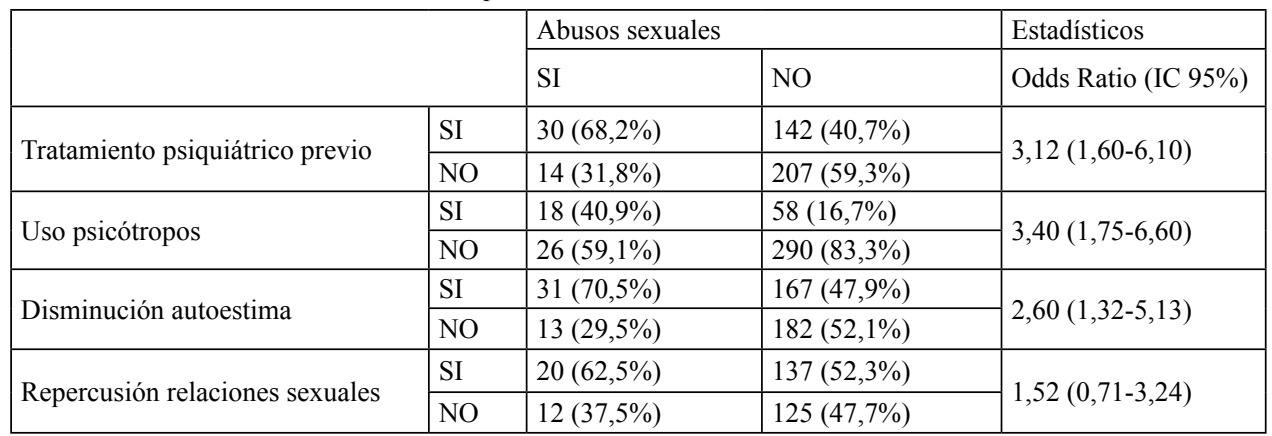

No se detectaron diferencias estadísticamente significativas $(p=0,607)$ en la proporción de pacientes que sienten vergüenza por su propio aspecto en las relaciones sexuales entre los que habían sufrido abusos sexuales y los que no.

\section{Discusión}

La prevalencia de abusos sexuales en nuestra muestra es análoga a otros estudios de obesos mórbidos con población americana (24). En estudios de prevalencia de abuso sexual realizados en España, describen que el 18,9\% de los adultos había sufrido abusos sexuales durante su infancia (25). Mientras que en nuestra muestra solo lo refieren el $11,1 \%$, esta diferencia probablemente corresponde a como se conceptualiza el abuso sexual (26). En el estudio de Pereda (27) se define el abuso en función de datos formales y en nuestra muestra consideramos como abusos sexuales solo los que el paciente refiere como tales en correspondencia con la vivencia subjetiva del hecho como erotizado e impuesto. 
Así mismo las características del abuso sexual en nuestra muestra no difieren de las encontradas en población general $(25,27)$.

Que el paciente refiera tener dificultades en las relaciones sexuales por la vergüenza de su aspecto, nos indica que considera que su imagen corporal de obeso interfiere en el acercamiento erótico con el otro y evidencia su convicción interna de que un cuerpo obeso no es sexualmente atractivo. Por lo que tomamos este ítem como índice de la capacidad de utilizar a nivel inconsciente la obesidad como protección ante la posibilidad de funcionar como reclamo sexual. Se supone que los pacientes con antecedentes de abusos sexuales serían más reticentes al acercamiento erótico al otro, sin embargo en nuestro estudio la proporción de este ítem es similar en los que han tenido abusos sexuales y en los que no.

Sí en cambio, es mucho mayor la muy baja autoestima y la consideración de la obesidad como causa de ella así como la probabilidad de haber precisado tratamiento psiquiátrico previo en los que han sido objeto de abusos sexuales. Los pacientes que han sido objeto de abuso sexual tienden a sentir su autoestima disminuida por ser obesos, es decir, viven su obesidad como algo que les resta valor personal, sin embargo no parece que sientan de forma significativa su obesidad como algo que les resta valor como reclamo erótico, al menos no más que aquellos que no han sufrido abusos sexuales.

En nuestra muestra de obesos mórbidos, la prevalencia de comorbilidad psiquiátrica en pacientes con antecedentes de abuso sexual es significativamente superior, lo que es coherente con el efecto traumático del abuso sexual. (28-32).

Si bien es cierto que los niños tienen una sexualidad y no hace falta la intervención del adulto para despertarla (30), las cualidades de la sexualidad infantil y la del adulto son muy diferentes. No es lo mismo que el niño descubra por si mismo en la interacción con el entorno su propia sexualidad que la estimulación forzada de una excitación inadecuada y excesiva para la capacidad del cuerpo y psiquismo en desarrollo del infante de contenerla y elaborarla.

Además el hecho de ser utilizado en la infancia como objeto de deseo por un adulto para su satisfacción erótica, conlleva en el infante la interiorización en su psiquismo en formación de una imagen esencialmente (global, general...) desvalorizada de sí mismo. Así la consideración de sí mismo como sujeto se ve trastocada. Tiene en mayor o menor medida una vivencia de sí mismo como "objeto" de deseo (lo que se percibe en la clínica como sentimientos de indignidad, suciedad, inadecuación etc....) y no como "sujeto" de deseo, que sería dueño de sí mismo de su cuerpo y sus deseos. "Alguien" y no "Algo" al servicio del deseo del otro.

En los casos en que el abuso sexual se ha realizado por parte de personas que son figuras de protección, la representación interna de dichas figuras (p.ej. padres) como peligrosas e inconsistentes resulta, con frecuencia, intolerable, por lo que esa vivencia es negada y disociada y en un intento de buscar explicaciones al abuso, 
que salvaguarde a "bondad" de la figura de protección asumen la carga de la culpa interiorizando la convicción de que ellos son esencialmente malos o indignos y merecedores por tanto del trato recibido $(28,30,32)$.

Además es muy difícil que las víctimas de abusos sexuales (y no sexuales) adquieran un sentimiento interno básico de seguridad, por lo que tienden a ser más dependientes de fuentes externas de confort. Una de esas fuentes puede ser la comida y la obesidad consecuente, una forma de expresar y perpetuar esa convicción de indignidad.

Entrando así en una espiral que se retroalimenta a sí misma y evoluciona hacia la obesidad mórbida. En los obesos mórbidos con antecedentes de abuso sexual es muy frecuente la baja autoestima y el sentimiento de indignidad atribuido a la obesidad. Sin embargo, de acuerdo con nuestros resultados, no parece que sientan su obesidad como algo que les resta valor específicamente como reclamo erótico, al menos no más que aquellos que no han sufrido abusos sexuales, ya que la proporción en ambos casos es similar. Lo que concuerda con los hallazgos encontrados por Oppong et al. (23) en obesos mórbidos intervenidos.

El que los obesos mórbidos refieran baja autoestima por obesidad implica que han interiorizado la imagen social denigrada del obeso, es decir, viven su obesidad como algo que les resta valor personal. Lo que, en nuestro estudio, es llamativamente superior en los pacientes que han sufrido abuso sexual en su infancia. Consideramos que es consecuencia de que los pacientes que han sufrido abusos sexuales interiorizan una imagen desvalorizada de si mismos, por el hecho mismo del abuso, y secundariamente la atribuyen a la obesidad. Siendo en estos casos la atribución consciente a la obesidad como causa de la baja autoestima un movimiento psíquico secundario. La elección de la obesidad como justificación del escaso valor que el individuo se da a si mismo está muy favorecida por el imaginario social (18).

\section{Conclusión}

Aunque es frecuente en los obesos la atribución a la obesidad de una mayor dificultad de acercamiento erótico al otro y la utilización de esta para evitar la erotización de las relaciones interpersonales no lo es más en los que han sido objeto de abuso sexual que en los que no lo han sido.

Los abusos sexuales aumentan de forma significativa la probabilidad de comorbilidad psiquiátrica, pero no predispone necesariamente a que se use el cuerpo como barrera de defensa ante las relaciones íntimas.

Los obesos mórbidos que han sufrido abusos sexuales en la infancia tienden a tener un sentimiento básico de indignidad e inadecuación (baja autoestima) y a considerar como causa de ese sentimiento su imagen corporal de obeso. 
Se confirma que el abuso sexual infantil tiene en la estructuración del psiquismo en formación, un efecto mucho más devastador que la mera reticencia al establecimiento de relaciones interpersonales estrechas, más o menos erotizadas.

En los obesos mórbidos que no han sufrido abuso sexual, la repercusión de su imagen corporal en la disminución de su autoestima es significativamente menor que en los que han sido objeto de abuso.

Para confirmar que en los obesos mórbidos con antecedentes de abuso sexual la atribución de la causa de su sentimiento básico de indignidad a la obesidad es un movimiento psíquico secundario, sería interesante estudiar de forma comparativa la evolución de la autoestima con el cambio de la imagen corporal tras la intervención.

\section{Bibliografia:}

(1) Colin A, Ross MD. Psychodynamics of eating disorder Behavior in Sexual Abuse Survivors. American journal of psychotherapy; 2009; 63, 3, 211.

(2) Isidro ML, Álvarez P, Martinez T, Cordido F. Alteraciones neuroendocrinas en la obesidad. Rev Med Univ Navarra 2004; 48, 2: 24-29.

(3) Eugenio Arteaga U, Manuel Moreno G. El tejido graso como modulador endocrino: Cambios hormonales asociados a la obesidad. Rev Med Chile 2010; 138: 1294-1301.

(4) Rohde P, Ichikawa L, Simon GE, Ludman J, Linde JA, Jeffery RW and Operskalski BH. Associations of child sexual and physical abuse with obesity and depression in middle-aged women. Child Abuse Negl; 2008; 32: 878-887.

(5) Fuemmeler BF, Dedert E, McClernon FJ, Beckham JC. Adverse childhood events are associated with obesity and disordered eating: Results from a U.S. population-based survey of young adults. J Trauma Stress. 2009; 22: 329-333.

(6) Gilbert R, Widom CS, Browne K, Fergusson D, Webb E, Janson S. Burden and consequences of child maltreatment in high-income countries. Lancet 2009;373: 68-81.

(7) King TK, Clark MW, Pera V. History of sexual abuse and obesity treatment outcome. Addict Behav 1996; 21:283-290.

(8) Noll JG. Obesity risk for female victims of childhood sexual abuse: a prospective study. Pediatrics, 2007; 120: e61-e67.

(9) Vámosi M, Heitmann BL, Kyvik KO. The relation between an adverse psychological and social environment in childhood and the development of adult obesity: a systematic literature review. Obesity Reviews, 2010; 11: 177-184.

(10) Grilo CM, Masheb RM, Brody M, Toth C, Burke-Martindale CH, Rothschild BS. Childhood maltreatment in extremely obese male and female bariatric surgery candidates. Obesity Research. 2005;13:123.

(11) Williamson DF, Thompson TJ, Anda RF, Dietz WH, Felitti V. Body weight and obesity in adults and self-reported abuse in childhood. International Journal of Obesity. 2002; 26: 1075-1082.

(12) Greenfield EA, Marks NF. Violence from parents in childhood and obesity in adulthood: using food in response to stress as a mediator of risk. Soc Sci Med. 2009;68 : 791-798. 
(13) Alvarez J, Pavao J, Baumrind N, Kimerling R. The relationship between child abuse and adult obesity among California women. Am J Prev Med 2007; 33: 28-33.

(14) Midei AJ, Matthews KA. Interpersonal violence in childhood as a risk factor for obesity: a systematic review of the literature and proposed pathways. Obesity Reviews, 2011; 12: e159-e172.

(15) Anisman H, Zaharia MD, Meaney MJ, Merali Z. Do early-life events permanently alter behavioral and hormonal responses to stressors? Int J Dev Neuroscience, 1998; 16: 149-64.

(16) Midei AJ, Matthews KA, Bromberger JT. Childhood abuse is associated with adiposity in midlife women: Possible pathways through trait anger and reproductive hormones. Psychosomatic Med 2010; 72: 215-223.

(17) Martín-Martín V, Loredo-Abdalá A. Estado de nutrición en niños víctimas de abuso físico y de abuso sexual. Rev Invest Clin 201; 62: 524-531.

(18) Contreras J. La obesidad: Una perspectiva sociocultural. Form Contin Nutr Obes 2002; $5: 275-86$.

(19) Wadden TA, Stunkard AJ. Psychosocial Consequences of obesity and dieting. Obesity theory and therapy, 2on edition, 1993 New York: Chapter 10; 163-175.

(20) Brownell KD. Behavioural treatment in person vs by mail, obesity, females. Therapist and group contact as variables in the behavioural treatment of obesity. Journal of Consulting \& Clinical Psychology. 6; 46: 593-594.

(21) Glinski J, Wetzler S, Goodman E. The psychology of gastric bypass surgery. Obesity surgery $2001 ; 11: 581-8$ -

(22) Nässlund E, Kkal JG. Patient selection and physiology of gastrointestinal antiobesity operations. Surg cin N Am 2005; 85: 725-740.

(23) Oppong BA, Nickels MW, Sax HC. The impact of a history of sexual abuse on weight loss in gastric bypass patients. Psychosomatics 2006; 47: 108-11.

(24) Buser A, et al . Outcome following Gastric Bypass Surgery: Impact of Past Sexual Abuse. Obesity Surgery 2004; 14: 170-174.

(25) Redondo-Figuero C, Ortiz-Otero MR. El abuso sexual infantil. Boletín de la sociedad de pediatría de Asturias, Cantabria y Castilla y León, 2005.

(26) Pereda N. El espectro del abuso sexual en la infancia: definición y tipología. Revista de Psicopatología y Salud Mental del Niño y del Adolescente. 2010; 16: 69-78.

(27) Pereda N, Foros M. Prevalencia y características del abuso sexual infantil en estudiantes universitarios españoles. Chile Abuso y Neglect. 2007; 31: 417-426.

(28) Ferenczi S: Psicoanálisis Tomo IV. Confusión de lenguas entre los adultos y el niño. Espasa-Calpe 1980 Madrid; Capítulo IX;139-149.

(29) Wildes JE, Kalarchian, MA, Marcus, MD, Levine, MD, Courcoulas, AP. Childhood Maltreatment and Psychiatric Morbidity in Bariatric Surgery Candidates. Obes Surg. 2008;18: 306-313.

(30) Hazan S. El abuso sexual por una figura paterna:encuentro entre amor y trauma. Cuadernos de Psicoanálisis XXXVII. 2004; 3-4: 20-49.

(31) Berezin de Guiter J. Traumas precoces. Abuso sexual. Daño en la constitución del psiquismo infantil. Rev. De Psicoanalisis. 2000, Tomo LVII, 2: 405-432.

(32) Glocer L. Tauma, violencia sexual y relaciones de poder. Rev. De Psicoanalisis. 2005. Tomo LXII, 2: 291-304. 